\title{
Conhecimento sobre o HIV/Aids de pessoas em situação de rua
}

\author{
Knowledge about HIV/Aids to street people
}

\section{Conocimiento sobre VIH/Aids a la gente de la calle}

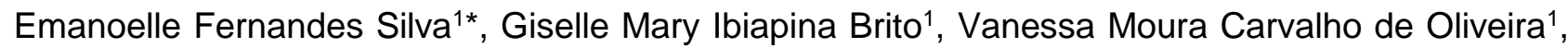
Matheus Sousa Marques Carvalho ${ }^{1}$, Braulio Vieira de Sousa Borges ${ }^{1}$, Rosilane de Lima Brito Magalhães ${ }^{1}$.

\section{RESUMO}

Objetivo: Avaliar o conhecimento sobre o HIV/Aids das pessoas em situação de rua. Métodos: Estudo transversal, realizado no Centro de Referência Especializado para População em Situação de Rua (Centro POP), em uma capital do Nordeste brasileiro, no período de agosto de 2018 a fevereiro de 2019, por meio de amostragem não probabilístico por conveniência. Aplicou-se o instrumento HIV Knowledge Questionnaire (HIV-K-Q). Foi realizado variância e alfa de Cronbach e teste qui quadrado para associação. Resultados: 50 pessoas em situação de rua foram abordadas, sendo a maioria do sexo masculino (86\%), a idade mediana foi de 35 anos e $(56 \%)$ possuía ensino fundamental completo. A população em situação de rua estudada, apresentou baixo conhecimento correto sobre o HIV, onde apenas 10 itens avaliados atingiram $70 \%$ de acertos, esse conhecimento esteve associado as variáveis faixa etária e estado civil. Conclusão: A população estudada apresenta baixo conhecimento sobre o HIV. Ser adulto jovem e solteiro esteve associado ao baixo conhecimento correto. Urge a realização de orientações sobre a prevenção do HIV e outras infecções para essa população.

Palavras-Chave: Conhecimento, HIV. Pessoas em situação de rua.

\section{ABSTRACT}

Objective: To evaluate the knowledge about VIH/SIDA of homeless people. Methods: A cross - sectional study was carried out at the Center of Specialized Reference for Population in Situation of the Street (POP Center), in a capital city of Northeast Brazil, from August 2018 to February 2019, through non - probabilistic sampling for convenience. The VIH Knowledge Questionnaire (VIH-K-Q) instruments was applied. Variance and alpha of Cronbach and chi-square test were performed for association. Results: 50 homeless people were approached, the majority being male $(86 \%)$, the median age was 35 years and $(56 \%)$ had complete elementary education. The population studied had low correct knowledge about $\mathrm{VIH}$, where only 10 items evaluated reached $70 \%$ of correct answers, this knowledge was associated with the variables age range and marital status. Conclusion: The population studied has low knowledge about $\mathrm{VIH}$. Being young and single adult was associated with low correct knowledge. There's an urgent need for guidelines on VIH prevention and other infections for this population.

Key words: Knowledge, HIV, Homeless Persons.

\section{RESUMEN}

Objetivo: Evaluar el conocimiento sobre el VIH / SIDA de las personas sin hogar. Métodos: Estudio transversal realizado en el Centro de Referencia Especializado para Población en Situación de Calle (Centro POP) en una capital del Nordeste brasileño, en el período de agosto de 2018 a febrero de 2019, por medio

${ }_{1}^{1}$ Universidade Federal do Piauí (UFPI), Teresina-PI. * E-mail: emanoellefernandes@hotmail.com. 
de muestra no probabilística por conveniencia. Se aplicó el instrumento VIH Knowledge Questionnaire (VIH$\mathrm{K}-\mathrm{Q})$. Se realizó la varianza y alfa de Cronbach y la prueba de qui cuadrado para la asociación. Resultados: 50 personas sin hogar fueron abordadas, siendo la mayoría del sexo masculino (86\%), la edad mediana fue de 35 años y (56\%) poseía enseñanza fundamental completa. Los indigentes estudiados, presentaran bajo conocimiento correcto sobre el VIH, donde sólo 10 ítems evaluados alcanzaron el $70 \%$ de aciertos, ese conocimiento estuvo asociado a las variables grupo de edad y estado civil. Conclusión: La población estudiada presenta bajo conocimiento sobre el VIH. Ser adulto joven y soltero estuvo asociado al bajo conocimiento correcto. Urge la realización de orientaciones sobre la prevención del VIH y otras infecciones para esa población.

Palabras clave: Conocimiento, VIH, Personas sin Hogar.

\section{INTRODUÇÃO}

Mesmo depois de mais de quatro décadas o Vírus da Imunodeficiência Humana (HIV) representa um grande desafio e preocupação para a saúde pública mundial, quando o número de infecções foi em média 1,8 milhões de novos casos no ano de 2016 (UNAIDS, 2016). Diante da magnitude do problema no mundo, o HIV tem sido apresentado com prevalências mais elevadas em populações chaves incluindo, Homens que fazem Sexo com Homens (HSH), usuários de drogas, profissionais do sexo e pessoas em situação de rua que varia de 8,2 a 32\% (SOARES JP, et al., 2017; BERBESÍ D, et al., 2015).

Dessa forma, torna-se motivo de preocupação considerando que a população em situação de rua é crescente no mundo, predominantemente masculina $81,3 \%$, com média de idade de 36 anos e baixa escolaridade, que vive entre a exclusão social e a falta de integração, atingindo um padrão de marginalização da sua condição. A falta de acesso ao serviço de saúde associado à falta de conhecimento aumenta a vulnerabilidade aos diversos agravos que estão expostos, com destaque para as Infecções Sexualmente Transmissíveis (IST), HIV e Síndrome da Imunodeficiência Adquirida (Aids) (CARVALHO PMRS, et al., 2017).

Estudo realizado em Los Angeles (Califórnia) com 421 pessoas em situação de rua mostrou que 64\% dos entrevistados relataram ter realizado testes de HIV há menos de um ano e $40 \%$ relataram testes para outras IST. A positividade para o HIV foi autorreferida em 10\%. Entre as pessoas que eram HIV-positivas, $57,1 \%$ relataram adesão parcial ao tratamento antirretroviral (WENZEL SL, et al., 2017).

Segundo pesquisadores, pessoas em situação de rua, possuem ideias equivocadas sobre as medidas de prevenção do HIV/Aids, associado a insuficiência das redes de apoio, preconceito, frequência do uso de drogas e não uso de preservativo e isso aumenta a exposição a novas infecções (FERNÁNDEZ DB, et al., 2017).

Com base no exposto, saber o conhecimento sobre o HIV nessa população torna-se importante considerando a falta de informação e práticas de risco que podem ampliar a vulnerabilidade ao HIV. Dessa forma objetivou-se avaliar o conhecimento sobre HIV/Aids das pessoas que vivem em situação de rua.

\section{MÉTODOS}

Estudo analítico, transversal com abordagem quantitativa realizado com pessoas em situação de rua da zona central de uma capital do Nordeste brasileiro. A coleta de dados foi realizada em setembro 2017 a junho 2018 e os participantes foram abordados em uma sala do Centro de Referência para Pessoas em Situação de rua (Centro POP). Adotou-se uma amostra não probabilística por conveniência, ressalvado o aspecto de que os resultados obtidos apresentam restrições a uma generalização ampla. Como critérios de inclusão considerou-se: ser de ambos os sexos; ter idade igual ou superior a 18 anos; ter no mínimo um atendimento pelo Centro POP; viver em situação de rua ou em apoio de albergues e não apresentar comportamento agressivo no momento da coleta de dados. Excluíram-se aqueles que se encontrava visivelmente sob influência de álcool e outras drogas no momento da coleta de dados. 
Os participantes responderam a um instrumento composto de duas partes. A primeira utilizou-se um formulário destinado à obtenção dos dados de caracterização (sexo, idade, estado civil e escolaridade), e a segunda, parte específica, utilizou-se um instrumento com 31 afirmações retiradas do HIV Knowledge Questionnaire (HIV-K-Q) (CAREY MP, MORRISON BD, JOHNSON BT, 1997).

Todos os participantes receberam orientações para classificar cada afirmativa como verdadeiro, falso ou não sei, por tratar-se de um questionário aplicado para pessoas alfabetizadas, saber ler e escrever constituiuse de critério de inclusão para o estudo.

Os dados foram coletados em sala específica para garantir o sigilo e privacidade dos participantes. Para a realização da segunda parte, os participantes foram organizados em grupos de no máximo 6 pessoas para aplicação do questionário sobre o conhecimento do HIV. Após a coleta de dados receberam orientações sobre medidas de prevenção para o HIV.

Os dados foram digitados em planilhas eletrônicas no Microsoft Office Excel for Windows 2013, por dupla digitação e, posteriormente, exportados e processados no Programa Statistical Package for the Social Sciences (SPSS) versão 21.00.

Foi realizado frequência, porcentagem, média, mediana e desvio padrão. Para verificar consistência interna foi verificado variância e a confiabilidade pelo alfa de Cronbach, que pode variar entre 0 e 1 sendo que os valores de referência são :>0,9 muito boa; 0,8 - 0,9 boa; 0,7 - 0,8 média; 0,6 - 0,7 razoável; 0,5 - 0,6 Má; $<0,5$ inaceitável (PESTANA MH, GAGEIRO JN, 2014). E foi realizado o teste qui quadrado para verificar associação.

O estudo atendeu todas as exigências formais seguindo às diretrizes da Resolução 466/12 do Conselho Nacional de Saúde do Ministério da Saúde. Com número do parecer: 1.755.893.

\section{RESULTADOS}

Do total de participantes $(n=50), 43(86 \%)$ era do sexo masculino e $42(84 \%)$ não possuíam companheiro. A idade mediana foi de 35 anos, com 29 (58\%) com idade entre 31 e 49 anos. Em relação à escolaridade 35 (70\%) possuem oito anos ou mais de estudos (Tabela 1). $O$ índice de consistência interna, alfa de Cronbach foi de 0,77 , sendo considerado razoável.

Tabela 1 - Variáveis sociodemográficas de pessoas que vivem em situação de rua, n=50. Teresina, PI, 2019.

\begin{tabular}{l|c|c}
\hline \multicolumn{1}{c|}{ Variáveis } & $\mathbf{N}$ & (\%) \\
\hline Sexo & 43 & 86 \\
Masculino & 7 & 14 \\
Feminino & & \\
\hline Faixa etária & 21 & 42 \\
Até 30 anos & 29 & 58 \\
$>31$ a 49 anos & & \\
\hline Estado civil & 8 & 16 \\
Tem companheiro & 42 & 84 \\
Não tem companheiro & & \\
\hline Escolaridade & 15 & 30 \\
$\leq 8$ anos de estudo & 35 & 70 \\
$>8$ anos de estudo & & \\
\hline
\end{tabular}

Fonte: Dados primários, 2019.

Uma frequência de resposta correta sobre o HIV foi verificada em $32 \%$ das afirmações, considerando um escore igual ou superior a 70\%, e essa prevalência esteve relacionada aos indivíduos maiores que 31 anos de idade e estarem solteiros. Destaca-se que $3(9,6 \%)$ estavam abaixo de $25 \%$ do escorre de respostas corretas (Tabela 2 e 3). 
Tabela 2 - Conhecimento correto sobre o HIV de pessoas em situação de rua, por faixa etária, $\mathrm{n}=50$. Teresina, $\mathrm{PI}, 2019$.

\begin{tabular}{|c|c|c|c|c|}
\hline \multirow{2}{*}{ Afirmações } & \multicolumn{2}{|c|}{$\begin{array}{l}\text { Faixa etária } \\
\text { (anos) }\end{array}$} & \multirow{2}{*}{$\begin{array}{l}\text { Resposta } \\
\text { correta } \\
\text { n (\%) }\end{array}$} & \multirow[t]{2}{*}{$\begin{array}{l}\text { Valor } \\
\text { de } p^{*}\end{array}$} \\
\hline & Até 30 & $>31$ & & \\
\hline Existe cura para aids & $13(26)$ & $21(42)$ & $34(68)$ & 0,282 \\
\hline Uma pessoa pode pegar HIV sentando-se no vaso sanitário & $10(20)$ & $16(30)$ & $26(52)$ & 0,965 \\
\hline Tosse e espirro não transmite o HIV & $9(18)$ & $8(16)$ & $17(34)$ & 0,446 \\
\hline O HIV pode ser transmitido por mosquitos & $13(26)$ & $16(32)$ & $29(56)$ & 0,635 \\
\hline Aids é causado pelo HIV & $18(36)$ & $20(40)$ & $38(76)$ & 0,194 \\
\hline $\begin{array}{l}\text { Uma pessoa pode pegar o HIV ao compartilhar um copo com } \\
\text { uma pessoa com HIV }\end{array}$ & $11(22)$ & $20(40)$ & $31(62)$ & 0,090 \\
\hline É possível pegar o HIV quando uma pessoa faz uma tatuagem & $17(34)$ & $23(46)$ & $40(80)$ & 0,285 \\
\hline $\begin{array}{l}\text { Uma pessoa pode pegar o HIV se fizer sexo anal com um } \\
\text { homem }\end{array}$ & $20(40)$ & $26(52)$ & $46(92)$ & 0,817 \\
\hline $\begin{array}{l}\text { Comer alimentos saudáveis impedem que uma pessoa pegue o } \\
\text { HIV }\end{array}$ & 11(22) & 19(42) & $30(60)$ & 0,666 \\
\hline $\begin{array}{l}\text { Todas as mulheres gravidas com HIV terão bebês que nascerão } \\
\text { com Aids }\end{array}$ & $8(16)$ & $7(14)$ & $15(30)$ & 0,044 \\
\hline Uma pessoa com HIV pode parecer e sentir-se saudável & 11(22) & 16(32) & $27(54)$ & 0,560 \\
\hline $\begin{array}{l}\text { As pessoas com HIV rapidamente mostram sérios sinais de } \\
\text { estarem com o vírus }\end{array}$ & $9(18)$ & $15(30)$ & $24(48)$ & 0,676 \\
\hline $\begin{array}{l}\text { Uma pessoa pode estar com HIV por } 5 \text { anos ou mais sem ter } \\
\text { Aids }\end{array}$ & $7(14)$ & $17(34)$ & $24(48)$ & 0,404 \\
\hline Existe uma vacina que impede as pessoas de pegarem o HIV & 11(22) & 16(32) & $27(54)$ & 0,108 \\
\hline Existem medicamentos para o tratamento da Aids & $18(36)$ & $25(50)$ & $43(86)$ & 0,638 \\
\hline $\begin{array}{l}\text { Mulheres são testadas para o HIV durante o exame preventivo } \\
\text { do câncer (Papanicolau) }\end{array}$ & $2(4)$ & $6(12)$ & $8(16)$ & 0,419 \\
\hline $\begin{array}{l}\text { Uma pessoa não pega o HIV por praticar sexo oral (boca no } \\
\text { pênis) em um homem com HIV }\end{array}$ & $7(14)$ & $10(20)$ & $17(34)$ & 0,850 \\
\hline Uma pessoa pode pegar o HIV ao doar sangue & $4(8)$ & $5(10)$ & $9(18)$ & 0,041 \\
\hline $\begin{array}{l}\text { Uma mulher não pega o HIV se fizer sexo durante a } \\
\text { menstruação }\end{array}$ & $7(14)$ & $9(18)$ & $16(32)$ & 0,041 \\
\hline $\begin{array}{l}\text { Existe uma camisinha feminina que ajuda a diminuir as chances } \\
\text { de uma mulher pegar o HIV }\end{array}$ & $19(38)$ & $26(52)$ & $45(90)$ & 0,572 \\
\hline Uma pessoa não pegara o HIV se estiver tomando antibióticos & $10(20)$ & $10(20)$ & $20(40)$ & 0,770 \\
\hline $\begin{array}{l}\text { Fazer o teste para HIV uma semana depois de fazer sexo dirá } \\
\text { se uma pessoa tem HIV }\end{array}$ & $8(16)$ & $12(24)$ & $20(40)$ & 0,720 \\
\hline $\begin{array}{l}\text { Uma pessoa pode pegar HIV ao entrar em uma piscina ou } \\
\text { banheira com alguém que tem HIV }\end{array}$ & 16(32) & $22(44)$ & $38(76)$ & 0,098 \\
\hline $\begin{array}{l}\text { Uma pessoa pode pegar o HIV através do contato com saliva, } \\
\text { lagrimas, suor ou urina }\end{array}$ & $12(24)$ & $14(28)$ & $26(52)$ & 0,540 \\
\hline $\begin{array}{l}\text { Uma pessoa pode pegar o HIV através das secreções vaginais } \\
\text { da mulher }\end{array}$ & $17(34)$ & $26(52)$ & $43(86)$ & 0,714 \\
\hline $\begin{array}{l}\text { Uma pessoa pode pegar o HIV se fizer sexo oral (boca na } \\
\text { vagina) em uma mulher }\end{array}$ & $15(30)$ & $21(44)$ & $36(72)$ & 0,892 \\
\hline $\begin{array}{l}\text { Utilizar vaselina ou óleo de bebe na camisinha diminui a chance } \\
\text { de pegar o HIV }\end{array}$ & $11(22)$ & $14(28)$ & $25(50)$ & 0,478 \\
\hline $\begin{array}{l}\text { A lavagem com água fria do material utilizado no uso de drogas } \\
\text { mata o HIV }\end{array}$ & $11(22)$ & $24(48)$ & $35(70)$ & 0,121 \\
\hline $\begin{array}{l}\text { Se uma pessoa tiver um teste positivo para o HIV, o local onde o } \\
\text { teste foi feito terá que avisar todos seus respectivos parceiros } \\
\text { sexuais }\end{array}$ & $3(6)$ & $8(16)$ & $11(22)$ & 0,088 \\
\hline $\begin{array}{l}\text { Pessoas que utilizam anabolizantes e esteroides injetáveis } \\
\text { podem pegar HIV ao compartilhar as agulhas }\end{array}$ & $18(36)$ & $26(52)$ & $44(88)$ & 0,462 \\
\hline Tomar vitaminas evita que uma pessoa pegue o HIV & $13(26)$ & $21(42)$ & $34(68)$ & 0,634 \\
\hline
\end{tabular}

Fonte: Dados primários, 2019.

Legenda: $\mathrm{p}^{*}$ (Teste Qui-Quadrado). 
Tabela 3 - Conhecimento correto sobre o HIV de pessoas em situação de rua considerando o estado civil e escolaridade, n=50. Teresina, PI, Brasil, 2019.

\begin{tabular}{|c|c|c|c|c|c|c|c|c|}
\hline \multirow[t]{2}{*}{ Afirmações } & \multicolumn{2}{|c|}{ Tem companheiro } & \multirow{2}{*}{$\begin{array}{l}\text { Resposta } \\
\text { correta } \\
\text { n (\%) }\end{array}$} & \multirow{2}{*}{$\begin{array}{l}\text { Valor de } \\
p^{\star}\end{array}$} & \multicolumn{2}{|c|}{ Escolaridade (anos) } & \multirow{2}{*}{$\begin{array}{l}\text { Resposta } \\
\text { correta } \\
\text { n (\%) }\end{array}$} & \multirow{2}{*}{$\begin{array}{l}\text { Valor de } \\
\mathbf{p}^{*}\end{array}$} \\
\hline & Sim & Não & & & $\leq 8$ & $>8$ & & \\
\hline Existe cura para aids & $3(6)$ & $31(62)$ & $34(68)$ & 0,029 & $9(18)$ & $23(46)$ & $34(68)$ & 0,808 \\
\hline $\begin{array}{l}\text { Uma pessoa pode pegar HIV sentando-se no } \\
\text { vaso sanitário }\end{array}$ & $3(6)$ & $23(46)$ & $26(52)$ & 0,367 & $5(10)$ & $21(42)$ & $26(52)$ & 0,531 \\
\hline Tosse e espirro não transmite o HIV & $3(6)$ & $14(28)$ & $17(34)$ & 0,241 & $5(10)$ & $12(24)$ & $17(34)$ & 0,922 \\
\hline O HIV pode ser transmitido por mosquitos & $4(8)$ & $25(50)$ & $29(56)$ & 0,558 & $7(14)$ & $22(44)$ & $29(56)$ & 0,466 \\
\hline Aids é causado pelo HIV & $6(12)$ & $32(64)$ & $38(76)$ & 0,398 & $11(22)$ & $27(54)$ & $38(76)$ & 0,771 \\
\hline $\begin{array}{l}\text { Uma pessoa pode pegar o HIV ao } \\
\text { compartilhar um copo com uma pessoa com } \\
\text { HIV }\end{array}$ & $2(4)$ & $29(58)$ & $31(62)$ & 0,401 & $8(16)$ & $23(46)$ & $31(62)$ & 0,288 \\
\hline $\begin{array}{l}\text { É possível pegar o HIV quando uma pessoa } \\
\text { faz uma tatuagem }\end{array}$ & $6(12)$ & $34(68)$ & $40(80)$ & 0,594 & 12(24) & $28(56)$ & $40(80)$ & 0,335 \\
\hline $\begin{array}{l}\text { Uma pessoa pode pegar o HIV se fizer sexo } \\
\text { anal com um homem }\end{array}$ & $7(14)$ & $39(78)$ & $46(92)$ & 0,138 & $14(28)$ & $32(64)$ & $46(92)$ & 0,853 \\
\hline $\begin{array}{l}\text { Comer alimentos saudáveis impedem que } \\
\text { uma pessoa pegue o HIV }\end{array}$ & $2(4)$ & $28(56)$ & $30(60)$ & 0,147 & $4(8)$ & 11(22) & $30(60)$ & 0,802 \\
\hline $\begin{array}{l}\text { Todas as mulheres gravidas com HIV terão } \\
\text { bebês que nascerão com Aids }\end{array}$ & $2(4)$ & $13(26)$ & $15(30)$ & 0,306 & $6(12)$ & $21(42)$ & $15(30)$ & 0,489 \\
\hline $\begin{array}{l}\text { Uma pessoa com HIV pode parecer e sentir-se } \\
\text { saudável }\end{array}$ & $2(4)$ & $25(50)$ & $27(54)$ & 0,041 & $6(12)$ & $21(42)$ & $27(54)$ & 0,489 \\
\hline $\begin{array}{l}\text { As pessoas com HIV rapidamente mostram } \\
\text { sérios sinais de estarem com o vírus }\end{array}$ & $2(4)$ & $22(44)$ & $24(48)$ & 0,087 & $7(14)$ & 17(34) & $24(48)$ & 0,350 \\
\hline $\begin{array}{l}\text { Uma pessoa pode estar com HIV por } 5 \text { anos } \\
\text { ou mais sem ter Aids }\end{array}$ & $4(8)$ & $20(40)$ & $24(48)$ & 0,413 & $9(18)$ & $15(30)$ & $24(48)$ & 0,298 \\
\hline $\begin{array}{l}\text { Existe uma vacina que impede as pessoas de } \\
\text { pegarem o HIV }\end{array}$ & 2(4) & $25(50)$ & $27(54)$ & 0,017 & $7(14)$ & $20(40)$ & $27(54)$ & 0,467 \\
\hline $\begin{array}{l}\text { Existem medicamentos para o tratamento da } \\
\text { Aids }\end{array}$ & $6(12)$ & $37(74)$ & $43(86)$ & 0,424 & $15(30)$ & $28(56)$ & $43(86)$ & 0,389 \\
\hline $\begin{array}{l}\text { Mulheres são testadas para o HIV durante o } \\
\text { exame preventivo do câncer (Papanicolau) }\end{array}$ & $2(4)$ & $6(12)$ & $8(16)$ & 0,212 & $7(14)$ & $10(20)$ & $8(16)$ & 0,797 \\
\hline $\begin{array}{l}\text { Uma pessoa não pega o HIV por praticar sexo } \\
\text { oral (boca no pênis) em um homem com HIV }\end{array}$ & $2(4)$ & 15(30) & $17(34)$ & 0,186 & $2(4)$ & $7(14)$ & 17(34) & 0,307 \\
\hline
\end{tabular}




\begin{tabular}{|c|c|c|c|c|c|c|c|c|}
\hline $\begin{array}{l}\text { Uma pessoa pode pegar o HIV ao doar } \\
\text { sangue }\end{array}$ & $2(4)$ & $7(14)$ & $9(18)$ & 0,185 & $2(4)$ & $7(14)$ & $9(18)$ & 0,307 \\
\hline $\begin{array}{l}\text { Uma mulher não pega o HIV se fizer sexo } \\
\text { durante a menstruarão }\end{array}$ & $3(6)$ & $13(26)$ & $16(32)$ & 0,995 & $8(16)$ & $8(16)$ & $16(32)$ & 0,244 \\
\hline $\begin{array}{l}\text { Existe uma camisinha feminina que ajuda a } \\
\text { diminuir as chances de uma mulher pegar o } \\
\text { HIV }\end{array}$ & $7(14)$ & $38(76)$ & $45(90)$ & 0,680 & $11(22)$ & $34(68)$ & $45(90)$ & 0,035 \\
\hline $\begin{array}{l}\text { Uma pessoa não pegara o HIV se estiver } \\
\text { tomando antibióticos }\end{array}$ & $2(4)$ & 18(36) & $20(40)$ & 0,653 & $7(14)$ & $13(26)$ & $20(40)$ & 0,790 \\
\hline $\begin{array}{l}\text { Fazer o teste para HIV uma semana depois de } \\
\text { fazer sexo dirá se uma pessoa tem HIV }\end{array}$ & $2(4)$ & $18(34)$ & $20(40)$ & 0,521 & $5(10)$ & $15(30)$ & $20(40)$ & 0,196 \\
\hline $\begin{array}{l}\text { Uma pessoa pode pegar HIV ao entrar em } \\
\text { uma piscina ou banheira com alguém que tem } \\
\text { HIV }\end{array}$ & $4(8)$ & $34(68)$ & $38(76)$ & 0,295 & $10(20)$ & $28(56)$ & $38(76)$ & 0,382 \\
\hline $\begin{array}{l}\text { Uma pessoa pode pegar o HIV através do } \\
\text { contato com saliva, lagrimas, suor ou urina }\end{array}$ & $3(6)$ & $23(46)$ & $26(52)$ & 0,473 & $7(14)$ & $19(38)$ & $26(52)$ & 0,902 \\
\hline $\begin{array}{l}\text { Uma pessoa pode pegar o HIV através das } \\
\text { secreções vaginais da mulher }\end{array}$ & $7(14)$ & $36(72)$ & $43(86)$ & 0,101 & $12(24)$ & $31(62)$ & $43(86)$ & 0,264 \\
\hline $\begin{array}{l}\text { Uma pessoa pode pegar o HIV se fizer sexo } \\
\text { oral (boca na vagina) em uma mulher }\end{array}$ & $6(12)$ & $31(62)$ & $36(72)$ & 0,410 & $13(26)$ & $24(48)$ & $36(72)$ & 0,156 \\
\hline $\begin{array}{l}\text { Utilizar vaselina ou óleo de bebe na camisinha } \\
\text { diminui a chance de pegar o HIV }\end{array}$ & $3(6)$ & $22(44)$ & $25(50)$ & 0,478 & $7(14)$ & $18(36)$ & $25(50)$ & 0,887 \\
\hline $\begin{array}{l}\text { A lavagem com água fria do material utilizado } \\
\text { no uso de drogas mata o HIV }\end{array}$ & $2(4)$ & $33(66)$ & $35(70)$ & 0,015 & 10(20) & $25(50)$ & $35(70)$ & 0,544 \\
\hline $\begin{array}{l}\text { Se uma pessoa tiver um teste positivo para o } \\
\text { HIV, o local onde o teste foi feito terá que } \\
\text { avisar todos seus respectivos parceiros } \\
\text { sexuais }\end{array}$ & 0 & $11(22)$ & $11(22)$ & 0,136 & $4(8)$ & $7(14)$ & $11(22)$ & 0,855 \\
\hline $\begin{array}{l}\text { Pessoas que utilizam anabolizantes e } \\
\text { esteroides injetáveis podem pegar HIV ao } \\
\text { compartilhar as agulhas }\end{array}$ & $6(12)$ & $38(76)$ & $44(88)$ & 0,019 & $14(28)$ & $30(60)$ & $44(88)$ & 0,059 \\
\hline $\begin{array}{l}\text { Tomar vitaminas evita que uma pessoa pegue } \\
\text { o HIV }\end{array}$ & $3(6)$ & $31(62)$ & $34(68)$ & 0,005 & $10(20)$ & $24(48)$ & $34(68)$ & 0,900 \\
\hline
\end{tabular}

Fonte: Dados primários, 2019.

Legenda: $\mathrm{p}^{*}$ (Teste Chi-Quadrado). 


\section{DISCUSSÃO}

Neste estudo, pessoas em situação de rua apresentam baixo conhecimento sobre HIV/Aids. Apenas 10 alcançaram o escore de acerto igual ou superior a $70 \%$ e três afirmações obtiveram menos de $25 \%$ de acertos (Tabelas 2 e 3). Corroboram com esses achados, pesquisa realizada em Sudão do Sul, nordeste da África, com 232 pessoas em situação de rua ao utilizarem o HIV K-Q, obteve-se conhecimentos razoável sobre o HIV (média=68.08\%) (FERREIRA AMN, 2016). O conhecimento avaliado não foi contemplado corretamente em todos os domínios, evidenciando que a população pesquisada apresenta baixo conhecimento sobre o HIV/Aids, o que acarreta maior vulnerabilidade a situações de risco de aquisição de infecção

Pessoas em situação de rua apresentam baixo conhecimento quanto a testagem, transmissibilidade por doação sanguínea e divulgação do diagnóstico sobre o HIV. É importante salientar que existe dentro dessa população uma reduzida associação entre a Aids como uma Infecção Sexualmente Transmissível (IST's), assim é recorrente que este público desconheça as formas de transmissão ou prevenção, sendo possível encontrar relatos como não tomar banho ou dormir no chão como formas de infecção pelo vírus (LUNA IT, et al., 2013). Nesse contexto, é válido perceber a carência de informações e conhecimento nesse público alvo, o que reforça a necessidade de educação em saúde a fim de promover saúde em todos os contextos e públicos diferenciados.

Considerando as vulnerabilidades da população que vive em situação de rua, infere-se que existe um risco de infecção pelo vírus do HIV, uma vez que, constantemente, estão inseridos em práticas de risco, tais fatores estão associados ao baixo conhecimento e habilidades escassas para acessar os serviços de saúde (NERLANDER LMC, 2015), A escassez de conhecimento sobre o tema aponta para uma atenção ao aumento das taxas de infecção nesse público, constituindo desta forma um fator de risco essencial a infecção por HIV.

As categorias que mais receberam respostas corretas, nesta pesquisa, foram em sua maioria relacionadas a transmissão, principalmente pela via sexual desprotegida, tratamento e formas de prevenção (Tabelas $2 \mathbf{e}$ 3). Estudo realizado com pessoas em situação de rua no Irã $(n=593)$ encontrou que mais de $90 \%$ dos participantes sabiam que o compartilhamento de agulhas é um método de transmissão do HIV e $93 \%$ dos participantes sabiam da possibilidade de transmissão do HIV através de sexo desprotegido entre homens (AMIRI FB, et al., 2018). Assim, o conhecimento da infecção pelo HIV/Aids por meio do sexo é encontrado dentro da população em situação de rua, que reconhece, também, a importância do uso do preservativo como estratégia de prevenir o HIV e outras IST's (DESMENNU AT, TITILOYE MA, OWOAJE ET, 2018). Portanto, deve-se reforçar a importância de práticas sexuais protegidas, independente da via, bem como o reconhecimento dos fatores de risco para aquisição de infecções.

Entretanto, em outro estudo, realizado com moradores de rua em uma cidade na Espanha, quando os participantes foram questionados sobre suas atividades sexuais e a falta de uso do preservativo, muitos relataram ter consciência dos riscos, mas preferem manter relações desprotegidas, os que optaram por essa escolha estiveram associados a preservação da masculinidade e do vínculo amoroso (CALVO F, GIRALT C, TURRÓ O, 2016), logo a disseminação de conhecimento sobre HIV sozinha não constitui um veículo para redução da taxas de infecção se pensamentos e costumes equivocados prevalecem dentro dessa comunidade, dessa forma, mesmo compreendendo as formas de evitar a infecção pelo HIV, a prática pode determinar ou não a adesão as essas estratégias o que pode resultar em barreiras na prevenção da infecção.

Quanto as características sociodemográficos (Tabela 1), a grande maioria é o do sexo masculino, adultos jovens e escolaridade baixa. Achados em estudos com pessoas em situação de rua na Grécia, Irã e Costa Rica, corroboram (SYPSA V, et al., 2015; ZADEH AOT, et al., 2014) (HIDALGO JL, MENDEZ AM, SEGURA DS, 2013); isso mostra que a maioria das pessoas nessa condição são jovens adultos que necessitam de maiores cuidados, uma vez que representam uma parcela ativa da sociedade em comportamentos e práticas de risco ao HIV.

Pessoas em situação de rua adultas jovens e solteiras apresentaram melhor conhecimento correto sobre HIV/Aids (Tabela 2). No entanto, a literatura não evidenciou associação do conhecimento entre a idade e 0 estado civil, mas aponta que os homens possuem melhor escore de conhecimento correto $(80,42 \%)$ do que 
as mulheres $(68,75 \%)(p=0,001)$ e um conhecimento significativamente maior do HIV é detectado em pessoas em situação de rua com formação acadêmica $(p=0,02)$ (AMIRI FB, et al., 2018). Neste contexto, os fatores sociodemográficos podem influenciar ou não, de forma significativa, na prevalência do conhecimento correto nesta população.

Em relação ao nível de escolaridade (Tabela 3), também foram encontrados semelhanças entre o presente estudo e a literatura, estudo realizado com 244 entrevistados em situação de rua mostrou que $73,36 \%$ possuíam menos do que um diploma do ensino médio completo e em outro estudo realizado no Irã 40,7\% dos pesquisados eram analfabetos (PARKER RD, DYKEMA S, 2014; ZADEH AOT, et al., 2014). Dessa forma, a baixa escolaridade é uma condição presente nesta população e sugere ter influências com a situação de saúde desses indivíduos. No entanto, esta variável não apresentou associação com o conhecimento apontado pelos entrevistados.

É importante salientar que a forma como a informação sobre o HIV são difundidas por essa população, contribui para a sua exposição ou não ao vírus, visto que, a presença de informações equivocadas dentro dessa comunidade, principalmente em relação as formas de transmissão é presente, como é apontado em um estudo realizado em Portland (EUA), onde uma grande parte deste grupo identificou a contracepção sem barreira como meio efetivo de prevenção do HIV (LOGAN LJ, et al., 2013). Por meio desta desinformação foi possível perceber que a exposição a infecção depende de como essa população se percebe em tal situação, pois existe uma distância significativa entre o que os usuários classificam como risco e o que eles percebem do mesmo.

Por tratar-se de uma população de difícil acesso e que está constantemente em mudanças ofertar uma assistência contínua torna-se um grande desafio, logo todas as intervenções foram realizadas no momento da coleta dados, o que para os pesquisadores foi bastante produtivo, pois seguido a aplicação do questionário e o surgimento de dúvidas foi possível realizar uma ação educativa contribuindo, dessa forma, para a ampliação do conhecimento nesta população. Recomenda-se que novas pesquisas sejam desenvolvidas com pessoas que vivem em situação de rua com o objetivo de compreender a realidade em que eles vivem e oferecer intervenções que potencializam a promoção da saúde.

\section{CONCLUSÃO}

População em situação de rua apresentou baixo conhecimento sobre o HIV/AIDS. Ainda, ser pessoas em situação de rua adultos jovens e solteiros estiveram associados ao pouco conhecimento correto sobre HIV/Aids. Diante dos dados apresentados percebe-se que a presença de informações sobre formas de prevenção e promoção da saúde constitui uma ferramenta fundamental para evitar IST. Faz-se necessário ofertar informações que contribuam para a construção de estratégias que viabilizem a formação de um saber crítico e responsável que esteja à disposição dessa população.

\section{REFERÊNCIAS}

1. AMIRI FB, et al. Knowledge, Attitude, and Practices Regarding HIV and TB Among Homeless People in Tehran, Iran. Int J Health Policy Manag, 2018; 7(6): 549-555.

2. BERBESí $D$, et al. Prevalencia y factores asociados al vih en habitante de calle de la ciudad de Medellín, Colombia. Rev. Fac. Nac. Salud Pública. 2015; 33(2): 200-205.

3. CALVO F, GIRALT C, TURRÓ O. Tuberculosis, Virus de la Inmunodeficiencia Humana y Virus de la Hepatitis-C en población sin-hogar. Index Enferm, 2016; 25(4): 248-52.

4. CAREY MP, MORRISON-BEEDY D, JOHNSON BT. The HIV-Knowledge Questionnaire: Development and evaluation of a reliable, valid, and practical self-administered questionnaire. AIDS Behav, 1997; (1): 61-74.

5. CARVALHO PMRS, et al. Prevalence, risk factors and hepatitis B immunization: helping fill the gap on hepatites $B$ epidemiology among homeless people, Goiânia, Central Brazil. Cad. Saúde Pública, 2017; 33(7): 1-9.

6. DESMENNU AT, TITILOYE MA, OWOAJE ET. Behavioural risk factors for sexually transmitted infections and health seeking behaviour of street youths in Ibadan, Nigeria. Afr Health Sci, 2018; 18(1): 180-187. 
7. FERNÁNDEZ DB. et al. Índice de vulnerabilidad al VIH en población habitante de calle. Enferm. Glob, 2017; (16)46: 154-67.

8. FERREIRA AMN. Conhecimentos sobre VIH/Sida nos clientes de um hospital de Lakes State - Sudão do Sul. Dissertação (Mestrado em Enfermagem Comunitária) - Instituto Politécnico de Viseu. Escola Superior de Saúde de Viseu, Viseu, 2016; 121p.

9. HIDALGO JL, MENDEZ AM, SEGURA DS. Seroprevalencia de hepatitis B, C y VIH en indigentes em Costa Rica. Rev Costarr Salud Pública, 2013; (22)2: 113-118.

10. LOGAN L. J. et al. Correlates of HIV Risk Behaviors Among Homeless and Unstably Housed Young Adults. Public Health Reports, 2013; (128): 153-160.

11. LUNA IT, et al. Conhecimento e prevenção das doenças sexualmente transmissíveis entre os adolescentes em situação de rua. Cienc Cuid Saude; 2013, 12(2):346-355

12. NERLANDER LMC, et al. Behaviors Associated with a Risk of HIV Transmission From HIV-Positive Street Youth to Non-Street Youth in Ukraine. Sex Transm Dis, 2015; (42)9: 513-520.

13. NYAMATHI A, et al. Correlates of Hepatitis B Virus and HIV Knowledge among Gay and Bisexual Homeless Young Adults in Hollywood. Am J Mens Health, 2013; (7)1: 18-26.

14. PARKER RD, DYKEMA S. Differences in Risk Behaviors, Care Utilization, and Comorbidities in Homeless Persons Based on HIV Status. JANAC, 2014; (25)3: 214-223.

15. PESTANA MH, GAGEIRO JN. (2014). Descobrindo regressão: com a complementaridade do SPSS. Lisboa: Edições Silabo.

16. SOARES JP, et al. Prevalência e fatores de risco para o hiv/aids em populações vulneráveis: uma revisão integrativa de literatura. Arq. Catarin Med, 2017; (46)4:182-194.

17. SYPSA V, et al. Homelessness and Other Risk Factors for HIV Infection in the Current Outbreak Among Injection Drug Users in Athens, Greece. Am J Public Health, 2015; (105)1: 196-204.

18. UNAIDS. Estatísticas, 2016. Disponível em: http://unaids.org.br/estatisticas. Acesso em: 21 fev. 2019.

19. WENZEL SL, et al. Risk behavior and access to HIV/AIDS prevention services in a community sample of homeless persons entering permanent supportive housing. AIDS Care, 2017; (29)5: 570-4.

20. ZADEH AOT, et al. Prevalence of HIV infection and the correlates among homeless in Tehran, Iran. Asian Pac J Trop Biomed, 2014; (4)1: 65-8. 